\title{
ANTERIOR TRANSLOCATION OF THE RIGHT PULMONARY ARTERY AS THE INITIAL SURGICAL OPTION FOR A PREMATURE NEWBORN WITH SEVERE BRONCHOPULMONARY DYSPLASIA
}

\author{
Okan Yurdakök ${ }^{1}$, Murat Cicek ${ }^{1}$, Oktay Korun ${ }^{1}$, Serap Ergor ${ }^{2}$, Arif Selcuk ${ }^{3}$, Firat Altin ${ }^{1}$, \\ Filiz Ozyilmaz ${ }^{1}$, Emine Yilmaz ${ }^{1}$, Numan Aydemir ${ }^{1}$, and Ahmet Sasmazel ${ }^{1}$ \\ ${ }^{1}$ Istanbul Dr Siyami Ersek Thoracic and Cardiovascular Surgery Training and Research \\ Hospital \\ ${ }^{2}$ Bezmialem Vakif University \\ ${ }^{3}$ Gaziantep Cengiz Gokcek Kadin Dogum ve Cocuk Hastaliklari Hastanesi
}

July 11, 2021

\begin{abstract}
We hereby present a case report of an extremely preterm newborn with bronchopulmonary dysplasia (BPD), spending more than 4 months of his early life in the newborn intensive care unit (NICU). The uniqueness of this case report is the difference in the algorithm used for the treatment of BPD with regards to the family's preference and its successful outcome.

Anterior translocation of THE right pulmonary artery AS THE INITIAL SURGICAL OPTION FOR A PREMATURE NEWBORN WITH severe bronchopulmonary dysplasia

Okan Yurdakök MD1, Murat Çiçek MD1, Oktay Korun MD1, Serap Nur Ergor MD 2, Arif Selcuk MD3, Fırat Hüsnü Altın MD1, Filiz Ozyilmaz MD4, Emine Hekim Yilmaz MD 5, Numan Ali Aydemir MD1, Ahmet Şaşmazel MD1.

1. Dr Siyami Ersek Thoracic and Cardiovascular Surgery Training and Research Hospital, Department of Pediatric Cardiac Surgery, Istanbul, TR

2. Bezmialem University Medical Faculty, Department of Pediatrics, Newborn Pediatric Intensive Care Unit, Istanbul, TR

3. Gaziantep Cengiz Gokcek Obstetrics, Gynecology and Children's Hospital, Department of Pediatric Cardiac Surgery, Gaziantep, TR

4. Dr Siyami Ersek Thoracic and Cardiovascular Surgery Training and Research Hospital, Department of Anesthesiology, Istanbul, TR

5. Dr Siyami Ersek Thoracic and Cardiovascular Surgery Training and Research Hospital, Department of Pediatric Cardiology, Istanbul, TR
\end{abstract}

Short Title: Anterior Translocation of Right Pulmonary Artery for Bronchopulmonary Dysplasia

Keywords: Prematurity, Bronchopulmonary Dysplasia

Corresponding Author: Okan Yurdakok

Siyami Ersek Hastanesi, Tibbiye Caddesi, 
Uskudar, Istanbul

Email: okanyurdakok@gmail.com

Fax: +90 (216) 4189649

* The authors do not have any financial disclosures.

\section{ABSTRACT}

We hereby present a case report of an extremely preterm newborn with bronchopulmonary dysplasia (BPD), spending more than 4 months of his early life in the newborn intensive care unit (NICU). The uniqueness of this case report is the difference in the algorithm used for the treatment of BPD with regards to the family's preferences and its successful outcome.

\section{CASE REPORT}

A 37-year-old female, who was previously G2P1A1, had a pre-eclamptic pregnancy and received steroids 2 days prior to delivery and gave birth to a very low weight actively crying baby. Apgar score was 7-8 and the baby was immediately put-on nasal CPAP with a $\mathrm{FiO} 2$ of $40 \%$.

The 26 weeks premature newborn, which was weighing 760 grams at birth, had a rough time in the newborn intensive care unit (NICU). NICU specialist successfully managed the baby to survive and able to gain weight up to 3,5 kilograms at the end of 4 months.

In the first 30 days, the baby was stable on full enteral feeding and was followed on room air according to the BPD protocol ${ }^{1}$ but was put on temporary non-invasive ventilatory support during agitation episodes.

The first significant respiratory distress episode was on day \# 70; the patient was put on non-invasive ventilatory support after the right lung showed pneumonic infiltration and treated accordingly. While improving clinically, the patient was intubated after a severe bronchospasm on day \# 79. Blood gases were consistent with respiratory acidosis and the X-Ray was showing signs of Acute Respiratory Distress Syndrome (ARDS). The baby was extubated on Day \# 88 but followed with non-invasive respiratory support.

The repeated transthoracic echocardiograms' (TTE) did not show any congenital heart disease or pulmonary hypertension. The baby was on steroids according to the BPD protocol but was never able to breathe on room air again. The patient was restless in general and more symptomatic when crying or agitated.

On Day \# 105, the patient suddenly deteriorated when both lungs collapsed and reintubated. No pneumonic infiltration detected and was extubated 10 days later but still on continuous respiratory support. The echocardiogram showed enlarged right heart structures and pulmonary arteries due to the newly developed pulmonary hypertension and successfully treated with a maximum dose of selective type 5 phosphodiesterase inhibitor and a short course of milrinone infusion after pediatric cardiology consultation, but the pulmonary arteries remain dilated even after treatment.

The repeated worsening episodes without any source of infection or ARDS make the team to consider the trachea-bronchomalasia (TBM) as a possible diagnosis and a dynamic flexible bronchoscopy was performed. Two main bronchuses were showing significant malasia but when the probe was advanced distally, the left main bronchus was showing signs of compression from outside.

A contrasted Computerized Tomograhy (CT) scan was planned to rule out any vascular rings or mediastinal structures, causing compression and obstruction. The results were consistent with generalized consolidated and atelectatic areas on both lungs and clear of any mediastinal abnormalities except the enlarged pulmonary arteries, especially the right pulmonary artery compressing and almost blocking the left main bronchus (Picture 1).

After 4 months in NICU with invasive and non-invasive ventilatory support; the long-term ventilatory support and the tracheostomy options were discussed exclusively with the team and the family. The family did not agree with the chronic respiratory support therapy, including tracheostomy and the home ventilator setting 
and their main argument was predicting the difficulty in maintaining proper childcare at home and they have concerns about handling the ventilator. So, they decided to proceed with the surgical option if there were any available.

Pediatric cardiologists and surgeons in the same hospital suggested that aortopexy operation might be a solution to relieve the compression on the bronchial tree, which was resistant to all medical strategy; and their suggestion was also supported with the literature ${ }^{234}$. The patient was consulted to our service by the newborn intensivist for a second opinion.

We initially requested to see the CT scan, in case if it was not evaluated in the light of a pulmonary artery sling anomaly or other vascular ring anomalies, which is quite common for missing the cause of recurrent pulmonary infections and difficulty in feeding. But there were no anomalies detected as stated earlier in the report; significantly enlarged right pulmonary artery was almost completely obstructing the left main bronchus soon after the bifurcation.

The options for surgical treatment of BPDs include open aortopexy, or recently, thoracoscopic aortopexy, tracheal resection and reconstruction or external stabilization and more recently direct anterior and/or posterior tracheopexy ${ }^{5}$. During the investigations in children with BPDs, it is very important to search for vascular causes of tracheal collapse with a CT, because vascular compression can be treated first or simultaneously as this may resolve or mitigate the severity of the TM. The technique of aortopexy was first used by Filler et al. in 1976, and it was derived from the operation described by Gross in 1948 to treat the "innominate artery compression syndrome" . Aortopexy is reported to be effective for treating tracheomalacia of different origins and other pathological conditions as well ${ }^{34}$. The main issue in our case was basically the significantly enlarged pulmonary arteries and especially the right pulmonary artery compressing the left main bronchus. Even if we used the technique for a variety of different indications in the past, we were not sure if aortopexy will be the right choice to create enough space for the left main bronchus compressed by the enlarged pulmonary arteries.

When we evaluate our options; we thought that performing either a modified LeCompte maneuver or an anterior translocation of right pulmonary artery will be a better choice to address the true compression on the left main bronchus, since the main issue was the enlarged pulmonary arteries due to pulmonary hypertension, accompanying the BPD. In the literature search, we have reached a few reports of using similar techniques, but they were all performed concomitant to surgeries for repair of congenital heart defects ${ }^{67}$ or for the bronchial compressions that were presented as a complication of previously performed surgeries for intracardiac congenital heart or other kind of defects and repaired afterwards ${ }^{8}$. There were not any other reports available in literature search for application of anterior translocation of the pulmonary as the initial choice for treating the isolated BPD. And another aspect in the decision-making process was whether to perform a LeCompte maneuver or an anterior translocation of the right pulmonary artery for treating the disease $^{78}$. Performing a classic LeCompte maneuver will necessitate a cross (X) clamp application and arresting the heart, which might be necessary to perform, when there is an enlarged and elongated aorta that needs to be shortened and reconstructed to create more free space for the trachea bronchial tree in the mediastinum. Otherwise, when there is no congenital heart disease in addition to BPD and no need to open the heart chambers, then it seems like there is no need to put the baby on unnecessary risk. We thought the anterior translocation of the right pulmonary artery is a better choice, which can be applied on cardiopulmonary bypass, without the need for arresting the heart. The augmentation of the pulmonary artery with fresh pericardium during the anterior translocation also prevents further tension on the vessel.

The case and the treatment options were discussed in our hospital's pediatric cardiology and pediatric heart surgery conference. There were concerns as the surgical decompression by translocating the right pulmonary artery that was compressing the left main bronchus from outside may not be enough to relieve the obstruction on the probably severe bronchomalacia. There were also concerns about putting the premature newborn under the risks of cardiopulmonary bypass without any congenital heart defects might surpass the potential benefits to relieve the external pressure on the bronchus. But considering the choice of the family, finally, the team agreed to proceed with anterior translocation of right pulmonary artery. 


\section{SURGICAL TECHNIQUE}

The terms were discussed with the family in detail and surgical consent was obtained. Midline incision was followed by sternotomy, followed by subtotal thymectomy. After heparinization, high aortic and bicaval cannulation was made accordingly, in case of a need for a Cross clamp (X clamp). The cardiopulmonary bypass was initiated, but the patient was not cooled down. A patent Ductus Arteriosus was found and divided. The pulmonary arteries were significantly enlarged and were dissected to free up from all adhesions all the way distally close to the intra pulmonary bifurcation level. The right pulmonary artery is transected from the main pulmonary artery and the proximal site on the distal MPA was over sawn as two layers; one being horizontal mattress and the other as continuous over and over with $6 / 0$ polypropylene suture. The right pulmonary artery was brought anterior to the aorta and spatulated on the posterior wall. Meanwhile a medially based trap door was created from the remaining anterior wall of the main pulmonary artery and attached and sawn to the backwall of the spatulated right pulmonary artery and the rest of the anterior wall of the reconstructed pulmonary artery is augmented with a generous sized patient's own fresh pericardium, to prevent any possible upward compression of the ascending aorta to the newly reconstructed anteriorly translocated pulmonary artery. The patient is weaned from cardiopulmonary bypass gradually following the optimization of the volume status and the ventilatory parameters and closed back up in the routine fashion, transferred to the pediatric cardiac ICU uneventfully.

On the 2nd postoperative day, the patient is transferred from the pediatric cardiac ICU to a pediatric ICU in a different hospital, weaned from ventilator successfully in the following week. Had a CT scan which was showing the translocated pulmonary arteries, the unobstructed left bronchus and the anteriorly translocated right pulmonary artery (Picture 2). The patient was discharged one month after surgery and was doing well, gaining weight and no difficulty with breathing and feeding were reported at the 11th month clinic visit.

\section{COMMENT}

Advances in perinatal care have dramatically improved the survival of extremely preterm infants, but the incidence of bronchopulmonary dysplasia (BPD) has not changed over the past few decades. BPD remains the most common late morbidity of preterm birth, but many controversies persist regarding how to best define BPD, grade its severity, prevent and treat the disease ${ }^{9}$.

The newborns with BPDs exposed to intubation and mechanical ventilation remains high along with a continued high incidence of chronic respiratory failure and the subsequent development particularly severe chronic respiratory disease and have related comorbidities that persist throughout their NICU course and needing prolonged respiratory support, including mechanical ventilation and high inspired oxygen concentrations post-discharge.

The decision to commit an infant with sBPD to chronic ventilator support with the placement of a tracheostomy tube can be complex and difficult and must involve extensive discussions among care providers and family members. Tracheostomy placement is only part of an organized strategy to provide chronic ventilation for enhancement of better long-term outcomes, if the optimized circumstances can be arranged properly.

But if there is resistance to recovery despite all medical management measures and an indication for surgery is inevitable; we thought that this case report might be helpful to shed light for keeping an open mind to find solutions for problems by using and combining different field practices. Another important aspect is as being the first case reported to use this congenital heart surgery method as the first choice instead of the last resort for an isolated BPD patient without any other congenital cardiac anomaly. In our opinion, for selected cases, it may be preferable to use the direct removal of the pressuring source method like the translocation of pulmonary artery, which might help to create more space for the bronchial tree to heal and grow, instead of the collateral pressure relief approach like aortopexy.

\section{REFERENCES}


1. Abman SH, Collaco JM, Shepherd EG, et al. Interdisciplinary Care of Children with Severe Bronchopulmonary Dysplasia. J Pediatr . 2017;181:12-28.e1. doi:10.1016/j.jpeds.2016.10.082

2. Rijnberg FM, Butler CR, Bieli C, et al. Aortopexy for the treatment of tracheobronchomalacia in 100 children: A 10-year single-centre experience. Eur J Cardio-thoracic Surg . 2018;54(3):585-592. doi:10.1093/ejcts/ezy076

3. Gruszka A, Sachweh JS, Schnoering H, et al. Aortopexy offers surgical options for a variety of pathological tracheal conditions in paediatric patients. Interact Cardiovasc Thorac Surg . 2017;25(4):589-594. doi:10.1093/icvts/ivx163

4. Torre M, Carlucci M, Speggiorin S, Elliott MJ. Aortopexy for the treatment of tracheomalacia in children: Review of the literature.Ital J Pediatr . 2012;38(1). doi:10.1186/1824-7288-38-62

5. Fraga JC, Jennings RW, Kim PCW. Pediatric tracheomalacia. Semin Pediatr Surg . 2016;25(3):156-164. doi:10.1053/j.sempedsurg.2016.02.008

6. Yamaguchi M, Ohashi H, Hosokawa Y, Oshima Y, Tsugawa C, Kimura K. Surgical treatment of airway obstruction associated with congenital heart disease in infants and small children. Eur J Cardio-thoracic Surg . 1991;5(9):479-485. doi:10.1016/1010-7940(91)90144-9

7. Chang YH, Sung SC, Kim H, Choo KS, Lee HD, Park JA. Anterior translocation of the right pulmonary artery to avoid airway compression in aortic arch repair. Ann Thorac Surg . 2013;96(6):2198-2202. doi:10.1016/j.athoracsur.2013.06.079

8. Greene CL, Mainwaring RD, Sidell D, Palmon M, Hanley FL. Lecompte Procedure for Relief of Severe Airway Compression in Children With Congenital Heart Disease. World J Pediatr Congenit Hear Surg . 2019;10(5):558-564. doi:10.1177/2150135119860466

9. Stoll BJ, Hansen NI, Bell EF, et al. Trends in care practices, morbidity, and mortality of extremely preterm Neonates, 1993-2012.JAMA - J Am Med Assoc . 2015;314(10):1039-1051. doi:10.1001/jama.2015.10244

Picture 1: A-coronal and B-transverse CT images with white arrows showing the narrowed and almost blocked left bronchus and the enlarged right pulmonary artery compressing from outside.

Picture 2: A-coronal and B-transverse CT images with black arrows showing the open left bronchus and the anteriorly translocated right pulmonary artery.

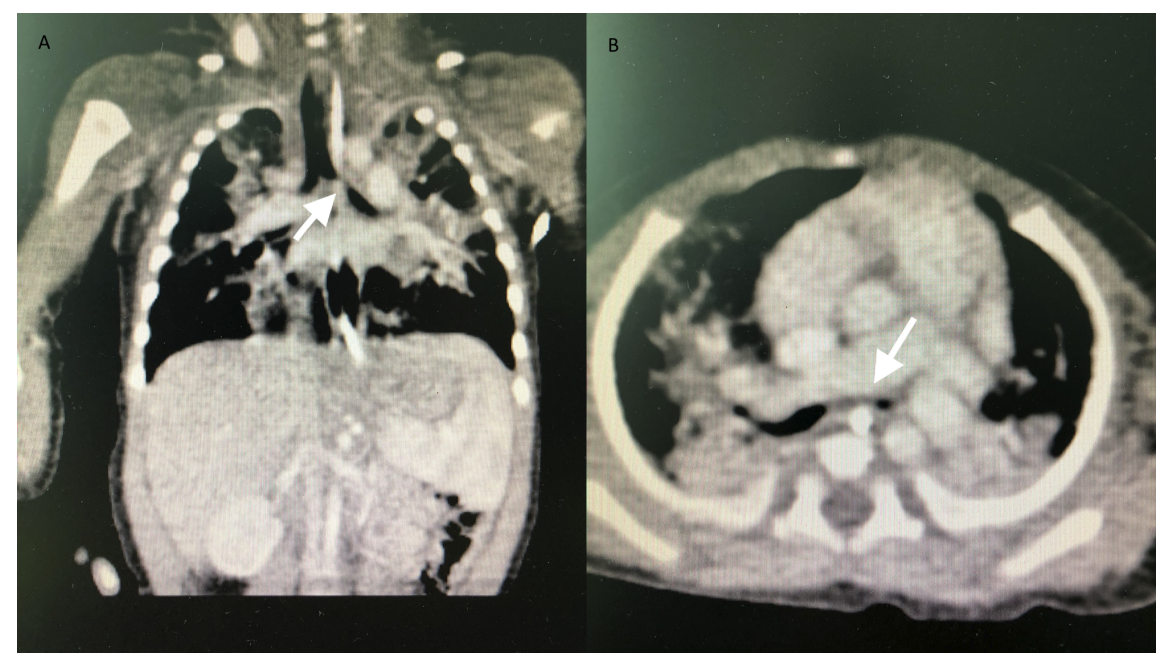



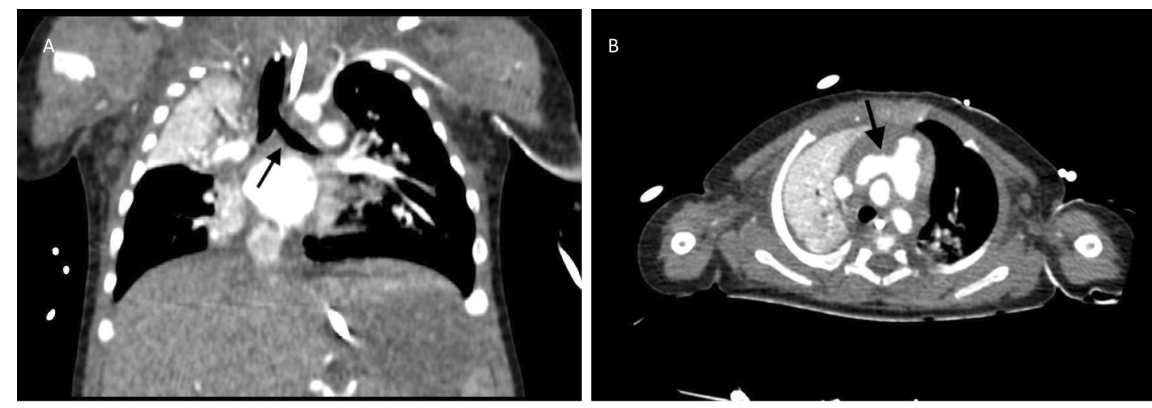